\title{
NONNEGATIVELY CURVED SUBMANIFOLDS IN CODIMENSION TWO
}

\author{
MARIA HELENA NORONHA
}

\begin{abstract}
Let $M$ be a complete noncompact manifold with nonnegative sectional curvatures isometrically immersed in Euclidean spaces with codimension two. We show that $M$ is a product over its soul, except when the soul is the circle $S^{1}$ or $M$ is 3-dimensional and the soul is the Real Projective Plane. We also give a rather complete description of the immersion, including the exceptional cases.
\end{abstract}

\section{INTRODUCTION}

Let $f: M^{n} \rightarrow \mathbb{R}^{n+2}$ be an isometric immersion of a complete Riemannian manifold $M$ with nonnegative sectional curvatures $(k \geq 0)$. If $M$ is compact, we have a topological classification of this manifold in [B-M $M_{1}$ and $\left.B-M_{2}\right]$. In this paper we want to consider the noncompact case. In [C-G], Cheeger and Gromoll have shown that a complete manifold with $k \geq 0$ is diffeomorphic to the total space of a vector bundle over a compact submanifold, its soul, and classified it in dimensions $\leq 3$, up to isometry. Then it is an interesting problem to know under which conditions $M$ turns out to be a product over its soul. In this paper show that this is always the case, except when the soul is the circle $S^{1}$ or the Real Projective Plane $\mathbb{R} P^{2}$. This is the content of Theorem 1 . We also give a rather complete description of the immersion, including these exceptional cases (Theorem $1(\mathrm{i})$ and Theorem 2 ). In a previous paper $\left[\mathrm{N}_{1}\right]$, we have some topological results. In another paper, $\left[\mathrm{N}_{2}\right]$, we prove that if there is a point $p \in M$ such that all the sectional curvatures are positive, then the soul of $M$ is a point, implying that $M$ is diffeomorphic to the Euclidean space $\mathbb{R}^{n}$.

Before stating our results, we recall that $f$ is called an $l$-cylindrical immersion if there exist the factorizations $M^{n}=M_{1}^{n-l} \times \mathbb{R}^{l}$ and $f=f_{1} \times i$, where $i: \mathbb{R}^{l} \rightarrow \mathbb{R}^{l}$ is the identity map. Then, in order to classify $M$ and $f$, we can suppose that $f$ is noncylindrical which is equivalent by Hartman $[\mathrm{H}]$ to the existence of a point $x$ in $M$ such that the index of relativity nullity $\nu(x)=0$, where

$$
\nu(x)=\operatorname{dim}\left\{X \in T_{x} M \mid \alpha(X, Y)=0, \forall Y \in T_{x} M\right\}
$$

Received by the editors January 2, 1990 and, in revised form, May 4, 1990.

1980 Mathematics Subject Classification (1985 Revision). Primary 53C40, 53C42.

Key words and phrases. Curvature operator, soul, product of immersions, index of relativity nullity.

Partially supported by FAPESP-SP-Brasil. 
and $\alpha$ is the second fundamental form. In what follows, $f$ will always be a noncylindrical immersion. Our first result states

Theorem 1. Let $f: M^{n} \rightarrow \mathbb{R}^{n+2}, n \geq 3$, be a noncylindrical isometric immersion of a complete noncompact manifold with nonnegative sectional curvatures and with an m-dimensional soul $A^{m}$. Then we have

(i) If $M$ is simply connected then $M$ is isometric to the product of a manifold homeomorphic to the sphere $S^{m}$ with a complete manifold $\mathscr{P}^{n-m}$ diffeomorphic to $\mathbb{R}^{n-m}$. If $n-m>1, f$ is a product of a hypersurface immersions.

(ii) If $M$ is not simply connected, the soul must be homeomorphic to the circle $S^{1}$ or to the Real Projective Space $\mathbb{R} P^{2}$. The latter case occurs only when $n=3$.

It follows by the theorem above that when $M$ is not simply connected, $M$ can be classified topologically. $M$ will be diffeomorphic:

(i) either to $S^{1} \times \mathbb{R}^{n-1}$ or to the total space of a nonorientable vector bundle over $S^{1}$.

(ii) either to $\mathbb{R} P^{2} \times \mathbb{R}$ or to total space of a nonorientable line bundle over $\mathbb{R} P^{2}$.

It also follows by Theorem 1 and the classification of compact manifolds immersed in codimension two that the immersion will be cylindrical over its soul when the soul is homeomorphic to a flat torus or a two-dimensional Klein bottle, or a product of two spheres, or the total space of a nonorientable fiber bundle over $S^{1}$.

When the codimension of the soul is 1 , we cannot expect to have a product of hypersurfaces in the Theorem 1, as we see in the following example: let $g: M_{1}^{n_{1}} \rightarrow \mathbb{R}^{n_{1}+1}$ be a codimension one isometric immersion and consider

$$
M_{1} \times \mathbb{R} \stackrel{g \times i}{\longrightarrow} \mathbb{R}^{n_{1}+2} \stackrel{h}{\longrightarrow} \mathbb{R}^{n_{1}+3}
$$

where $h$ is an isometric immersion. $f=h \circ(g \times i)$ is not in general either a product of immersions nor 1-cylindrical. For this case we prove

Theorem 2. Let $f: M^{n} \rightarrow \mathbb{R}^{n+2}$ be a noncylindrical isometric immersion, where $M=M_{1}^{n-1} \times \mathbb{R}$ is a simply connected manifold with nonnegative sectional curvatures and $n \geq 3$. Then

(i) There exists an isometric immersion $g$ from $M^{n-1}$ to $\mathbb{R}^{n}$.

(ii) Suppose that for each $x \in M$, there is a two-plane $\sigma \subset T_{x} M$ such that $k(\sigma)>0$; then $f$ is homotopic through isometric immersions to the immersion $g \times i: M=M_{1} \times \mathbb{R} \rightarrow \mathbb{R}^{n+1}$, where $i$ is the identity map.

Theorem 2 also covers the case of the universal covering of the manifolds which appear in Theorem 1(ii). We have not been able to construct a proof for Theorem 2(ii) without an extra condition for $k(\sigma)$. But we want to observe that the same statement can be proved if we do not require differentiability of the isometric homotopy.

Finally, by adding one extrinsic property to the immersion we are able to show that $f$ is always a product:

Theorem 3. With the same hypothesis of Theorem 1, suppose that the immersion has flat normal bundle $\left(\mathscr{R}^{\perp}=0\right)$. Then $M$ is a product over the soul $A$ and $f$ is a product of hypersurface immersions. It follows that the soul is homeomorphic to a sphere $S^{m}, m \geq 1$. 
At the end of $\S 5$ in this paper, modifying slightly the Yeaton Cliffon example which appears in [A-M], we see that we can have a complete noncompact manifold isometrically immersed in codimension two, which is a product over the soul $S^{1}$ but the immersion $f$ is not a product of hypersurface immersions.

In order to have a complete understanding of isometric immersions of nonnegatively curved manifolds in $\mathbb{R}^{n+2}$, it seems reasonable to ask if a manifold homeomorphic to $\mathbb{R} P^{2}$ with $k \geq 0$ can be isometrically immersed in $\mathbb{R}^{N}$ with the dimension of the first normal space at most two, since Gromov and Rokhlin in [G-R, p. 42] have proved that if $k>0, \mathbb{R} P^{2}$ cannot be embedded in $\mathbb{R}^{4}$.

Some of the research in this article was done while the author visited the University of California at Santa Barbara. We want to thank this department for its hospitality and especially Professor J. D. Moore for helpful discussions.

\section{Preliminaries AND REDUCIBILITY ALONG THE SOUL}

It is a well-known result of Weinstein [W], that if the codimension of an isometric immersion is two then the nonnegativity of the sectional curvatures $(k \geq 0)$ implies the nonnegativity of the curvature operator $(\mathscr{R} \geq 0)$.

For the case where $M^{n}$ is a complete noncompact manifold with $\mathscr{R} \geq 0$, we now collect some known results of a soul $A$ of $M$.

(2.1) A soul $A$ of $M$ is a compact, totally geodesic submanifold of $M$, without boundary and has $\mathscr{R} \geq 0$ (see $[\mathrm{C}-\mathrm{G}]$ ).

(2.2) If $M$ is simply connected, $M$ is isometric to the product $A^{M} \times \mathscr{P}^{n-m}$ where $\mathscr{P}^{n-m}$ is a complete manifold diffeomorphic to $\mathbb{R}^{n-m}$ (see $\left[\mathrm{N}_{2}\right]$ ).

(2.3) If $M$ is not simply connected, $M$ is locally isometric to a product over the soul $A$ (see $\left[\mathrm{N}_{2}\right]$ ).

In order to prove our theorems, we need the following concept.

(2.4) Definition. An isometric immersion $f: M^{n} \rightarrow \mathbb{R}^{n+p}, p \geq 1$, is called reducible along $A$ if for all $X \in T A$ and $Y \in T A^{\perp}$ we have $\alpha(X, Y)=0$.

As it is proved in [B-N], this property implies that $M$ is diffeomorphic to $A^{m} \times \mathbb{R}^{n-m}$. Another consequence of the reducibility along $A$ is given by the following proposition.

(2.5) Proposition. Let $f: M^{n} \rightarrow \mathbb{R}^{n+p}, p \geq 1$, be an isometric immersion of a complete noncompact manifold with nonnegative curvature operator. If $f$ is reducible along a soul $A$ then $M$ is isometric to a product $A^{k} \times \mathscr{P}^{n-k}$.

Proof. By (2.3) there is a Riemannian submersion $P: M \rightarrow A$ where the fibers, $F$ are totally geodesic. Hence it follows from [O] that to show that $M$ splits as a product, it is sufficient to prove that for each geodesic loop $\gamma:[0,1] \rightarrow A$, starting from a point $x \in A$, the map

$$
L_{\gamma}: \pi^{-1}(x)=F_{x} \rightarrow F_{x}, \quad L_{\gamma}(p)=\tilde{\gamma}(1),
$$

(where $\tilde{\gamma}$ denotes the horizontal lift of $\gamma$ with $\tilde{\gamma}(0)=p$ ) is the identity on $F_{x}$.

This will follow easily from the fact that $\tilde{\gamma}(0)=p$ belongs to the pseudo-soul $\bar{A}$ (see [Y]) obtained in the following way:

Consider $\sigma:[0, l] \rightarrow M$ the minimal unit speed geodesic connecting $p$ to the soul $A$. The vector $Y_{\sigma(l)}=-\sigma^{\prime}(l)$ is a normal vector of $A$. Since $f$ is 
reducible along $A$, we have

$$
\tilde{\nabla}_{X} Y=\nabla_{X} Y+\alpha(X, Y)=\nabla_{X} Y \text { for all } X \in T A
$$

(where $\tilde{\nabla}$ and $\nabla$ are the Riemannian connections of $\mathbb{R}^{n+p}$ and $M$ respectively) implying that the parallel translation of the vector $Y_{\sigma(l)}$ along $A$ in $\mathbb{R}^{n+p}$ gives a normal parallel section $Y$ along $A$.

Then the map $\varphi_{Y}: A \times \mathbb{R} \rightarrow M$, given by $\varphi_{Y}(x, t)=\exp _{x} t Y(x)$ is an isometric immersion (see [Y, Proposition 3.2], $\bar{A}=\varphi_{Y}(A, l)$ and $\tilde{\gamma}=\varphi_{Y}(\gamma, l)$.

\section{BASIC LEMMA AND PROOF OF THEOREM 1}

Let $r(x)$ be the Lie algebra generated by the range of the curvature operator $\mathscr{R}$ at the point $x$. Let $U$ be the orthogonal complement of the relative nullity subspace $N(x)$. Since the codimension of the immersion is 2 , by Theorem 1 in $[\mathrm{Bi}]$ we have the following possibilities for $r(x)$ :

(a) $r(x)=\theta(U)$

(b) $r(x)=\theta\left(V_{1}\right) \oplus \theta\left(V_{2}\right)$ where $V_{1} \oplus V_{2}=U$,

(c) there is a complex structure on $U$ and $r(x)$ is the unitary algebra of this structure (and the second fundamental forms are all of signature zero unless $\operatorname{dim} U=4)$,

where $\theta(U)$ is the exterior algebra of $U$. Moreover, if (b) occurs with $\operatorname{dim} V_{1}>$ 1 and $\operatorname{dim} V_{2}>1$ then $V_{1}$ and $V_{2}$ are orthogonal to each other and $R^{\perp}(x)=0$ where $R^{\perp}$ is the normal curvature tensor of $f$. (See Theorem $1\left(\mathrm{a}^{\prime}\right)$ of [Bi].)

We will use the classification above to prove the lemma below whose hypothesis is satisfied by our three theorems stated in $\S 1$. We will use the notation $A_{\xi}$ for the Weingarten operator, where $\xi$ is a normal vector.

(3.1) Lemma. Let $f: M^{n} \rightarrow \mathbb{R}^{n+2}$ be an isometric immersion. Suppose that there exists an open set $O$ of $M$ such that $O$ is a metric product $O_{1} \times O_{2}$. Then for $x \in 0$ we have

(a) If $\left.\mathscr{R}\right|_{\theta\left(T_{x} O_{i}\right)} \neq 0, i=1,2$, there is an orthonormal frame $\left\{\xi_{1}, \xi_{2}\right\}$ in the normal space such that $\left.A_{\xi_{1}}\right|_{T_{x} O_{2}}=0$ and $\left.A_{\xi_{2}}\right|_{T_{x} O_{1}}=0$.

(b) If $\left.\mathscr{R}\right|_{\theta\left(T_{x} O_{2}\right)}=0$ and $\alpha(Z, Z) \neq 0$ for some $Z \in T_{x} O_{2}$, there is an orthonormal frame $\left\{\xi_{1}, \xi_{2}\right\}$ such that $A_{\xi_{1}}\left(T_{x} O_{1}\right) \subseteq T_{x} O_{1}, A_{\xi_{1}}(Z)=0$ and rank $A_{\xi_{2}}=1$.

Proof. (a) The only possibility in Bishop's classification is $r(x)=\theta\left(V_{1}\right) \oplus \theta\left(V_{2}\right)$ with $\operatorname{dim} V_{1}>1$ and $\operatorname{dim} V_{2}>1$ whence $\mathscr{R}^{\perp}(x)=0$. Therefore, the conclusion follows by Bishop's Theorem.

(b) By hypothesis $\operatorname{dim} U \geq 1$. If $\operatorname{dim} U=1$ there exists a normal vector $\xi$ such that $A_{\xi}$ is identically null and then $\xi_{1}=\xi$ and $\xi_{2}$ orthogonal to $\xi$ satisfy the lemma.

If $\operatorname{dim} U=2$, we will define $\xi_{2}$ by $\alpha(Z, Z) /\|\alpha(Z, Z)\|$. Taking $\xi_{1}$ orthogonal to $\xi_{2},\left\langle A_{\xi_{1}} Z, Z\right\rangle=0$. But there exists a vector $Z_{1}$ which is the eigenvector of $A_{\xi_{1}}$ associated to its only nonnull eigenvalue. We claim that $Z_{1} \in T_{x} O_{1}$. In fact, $Z_{1}=a X+b Z$, where $X$ is the normalized orthogonal projection of $Z_{1}$ onto $T_{x} O_{1}$. If we take $Z_{1}^{\perp}=-b X+a Z, A_{\xi_{1}}\left(Z_{1}^{\perp}\right)=0$ then,

$$
0=-b A_{\xi_{1}} X+a A_{\xi_{1}} Z \text {. }
$$

Taking inner product with $Z$ (remembering that $\left\langle A_{\xi_{1}} Z, Z\right\rangle=0$ ) we have

$$
=0-b\left\langle A_{\xi_{1}} X, Z\right\rangle \text {. }
$$


If $b=0$ we have $Z_{1}=X$ and if $\left\langle A_{\xi_{1}} X, Z\right\rangle=0$ we have, because $A_{\xi_{1}}(Y)=$ 0 for $Y$ orthogonal to $X, Y \in T_{x} M_{1}$, that $T_{x} M_{1}$ is invariant by $A_{\xi_{1}}$ and $A_{\xi_{1}}(Z)=0$. Hence the lemma follows, for this case.

If $\operatorname{dim} U \geq 3$, it follows again by Bishop's classification that $r(x)$ is a nonnull algebra implying that $\left.\mathscr{R}\right|_{\theta\left(T_{x} O_{1}\right)} \neq 0$. We claim that the only possibility for $r(x)$ is $\theta\left(V_{1}\right) \oplus \theta\left(V_{2}\right)$ where $\operatorname{dim} V_{2}=1$. In fact, consider an orthonormal frame $\left\{X_{1}, \ldots, X_{n}\right\}$ of $T_{x} O$ such that $X_{1}, \ldots, X_{s} \in N(x)$ and $Z \in \operatorname{span}\left\{X_{1}, \ldots, X_{s}, X_{s+1}\right\}$. We have

$$
\mathscr{R}\left(X_{i} \wedge X_{j}\right)=0, \quad i=1, \ldots, s, j=1, \ldots, n .
$$

Denoting by $X^{\prime}$ and $X^{\prime \prime}$ the orthogonal projection of the vector $X$ onto $T_{x} O_{1}$ and $T_{x} O_{2}$ respectively, because the metric is a product, and $\mathscr{R} \mid T_{x} O_{2}=0$, we have

$$
\mathscr{R}\left(Z \wedge X_{j}\right)=\mathscr{R}\left(Z \wedge X_{j}^{\prime}\right)+\mathscr{R}\left(Z \wedge X_{j}^{\prime \prime}\right)=0, \quad j=1, \ldots, n .
$$

Then the range of $\mathscr{R}$ is contained in $\theta(W)$, where $W=\operatorname{span}\left\{X_{s+2}, \ldots, X_{n}\right\}$ which implies

$$
r(x) \subseteq \theta(n-s-1) .
$$

It is obvious that $r(x)$ cannot be $\theta(U)=\theta(n-s)$. If $n-s$ is even, $r(x)$ cannot be the unitary algebra $u((n-s) / 2)$, since $u((n-s) / 2)$ is not contained in $\theta(n-s-1)$. Then $r(x)=\theta(n-s-1) \oplus \theta(1)$ as we claimed.

Then, following the proof of Bishop's theorem, there is an orthonormal frame $\left\{\xi_{1}, \xi_{2}\right\}$ of the normal space such that the range of $A_{\xi_{1}}$ is $V_{1}$ and $\operatorname{rank} A_{\xi_{2}}=1$. We will need the calculation below to show that $V_{1} \subseteq T_{x} O_{1}$.

Since $\operatorname{rank} A_{\xi_{2}}=1$, the Gauss equation is reduced to

$$
\mathscr{R}=A_{\xi_{1}} \wedge A_{\xi_{1}} .
$$

Because $\operatorname{dim} n \geq 3, r(x)$ is a nonnull algebra, implying that there exist $X, Y \in$ $T_{x} M$ such that $\mathscr{R}(X \wedge Y) \neq 0$. Denoting again by $X^{\prime}$ and $X^{\prime \prime}$ the orthogonal projection on $T_{X} O_{1}$ and $T_{x} O_{2}$ respectively we have

$$
\begin{aligned}
\mathscr{R}(X \wedge Y)= & \left(A_{\xi_{1}} X\right)^{\prime} \wedge\left(A_{\xi_{1}} Y\right)^{\prime}+\left(A_{\xi_{1}} X\right)^{\prime} \wedge\left(A_{\xi_{1}} Y\right)^{\prime \prime} \\
& +\left(A_{\xi_{1}} X\right)^{\prime \prime} \wedge\left(A_{\xi_{1}} Y\right)^{\prime}+\left(A_{\xi_{1}} X\right)^{\prime \prime} \wedge\left(A_{\xi_{1}} Y\right)^{\prime \prime}
\end{aligned}
$$

where

and

$$
\omega_{1}=\left(A_{\xi_{1}} X\right)^{\prime \prime} \wedge\left(A_{\xi_{1}} Y\right)^{\prime \prime}=0
$$

$$
\omega_{2}=\left(A_{\xi_{1}} X\right)^{\prime} \wedge\left(A_{\xi_{1}} Y\right)^{\prime \prime}+\left(A_{\xi_{1}} X\right)^{\prime \prime} \wedge\left(A_{\xi_{1}} Y\right)^{\prime}=0,
$$

since the range of the curvature operator is contained in $\theta\left(T_{x} O_{1}\right)$.

Taking interior product of $\omega_{2}$ with $\left(A_{\xi_{2}} X\right)^{\prime}$ we get

$$
0=i\left(A_{\xi_{1}} X\right)^{\prime} \omega_{2}=\left\|\left(A_{\xi_{1}} X\right)^{\prime}\right\|^{2}\left(A_{\xi_{1}} Y\right)^{\prime \prime}-\left\langle\left(A_{\xi_{1}} Y\right)^{\prime},\left(A_{\xi_{1}} X\right)^{\prime}\right\rangle\left(A_{\xi_{1}} X\right)^{\prime \prime}
$$

and therefore

$$
\left(A_{\xi_{1}} Y\right)^{\prime \prime}=\left\langle\left(A_{\xi_{1}} Y\right)^{\prime},\left(A_{\xi_{1}} X\right)^{\prime}\right\rangle\left\|\left(A_{\xi_{1}} X\right)^{\prime}\right\|^{-2}\left(A_{\xi_{1}} X\right)^{\prime \prime} .
$$

Taking interior product with $\left(A_{\xi_{1}} Y\right)^{\prime}$ we get

$$
\begin{aligned}
0 & =i\left(A_{\xi_{1}} Y\right)^{\prime} \omega_{2}=\left\langle\left(A_{\xi_{1}} X\right)^{\prime},\left(A_{\xi_{1}} Y\right)^{\prime}\right\rangle\left(A_{\xi_{1}} Y\right)^{\prime \prime}-\left\|\left(A_{\xi_{1}} Y\right)^{\prime}\right\|^{2}\left(A_{\xi_{1}} X\right)^{\prime \prime} \\
& =\left\|\left(A_{\xi_{1}} X\right)^{\prime}\right\|^{-2}\left\{\left(\left\langle\left(A_{\xi_{1}} X\right)^{\prime},\left(A_{\xi_{1}} Y\right)^{\prime}\right\rangle\right)^{2}-\left\|\left(A_{\xi_{1}} X\right)^{\prime}\right\|^{2}\left\|\left(A_{\xi_{1}} Y\right)^{\prime}\right\|^{2}\right\}\left(A_{\xi_{1}} X\right)^{\prime \prime} .
\end{aligned}
$$


If $\left(A_{\xi_{2}} X\right)^{\prime \prime} \neq 0$ the above relation implies $\left(A_{\xi_{1}} Y\right)^{\prime}=\lambda\left(A_{\xi_{1}} X\right)^{\prime}$ and then $\mathscr{R}(X \wedge Y)=\left(A_{\xi_{1}} X\right)^{\prime} \wedge\left(A_{\xi_{1}} Y\right)^{\prime}=0$. Hence,

$$
\text { if } \mathscr{R}(X \wedge Y) \neq 0 \text {, we have }\left(A_{\xi_{1}} X\right)^{\prime \prime}=\left(A_{\xi_{1}} Y\right)^{\prime \prime}=0 \text {. }
$$

Consider now the orthonormal frame $\left\{Z_{1}, \ldots, Z_{n}\right\}$ which diagonalizes the operator $A_{\xi_{2}}$ such that $A_{\xi_{2}}\left(Z_{1}\right)=\lambda Z_{1}$ and $A_{\xi_{2}}\left(Z_{i}\right)=0, i \geq 2$. Since $\mathscr{R} \neq$ 0 at $x$, there exist $Z_{i}, Z_{j}$ such that $\mathscr{R}\left(Z_{i} \wedge Z_{j}\right) \neq 0$. By (3.3), we have $\left\langle\alpha\left(Z_{i}, Z\right), \xi_{1}\right\rangle=0=\left\langle\alpha\left(Z_{i}, Z\right), \xi_{2}\right\rangle=0$. This implies $\alpha\left(Z_{i}, Z\right)=0$ for $i \geq 2$, as we have supposed $A_{\xi_{2}}\left(Z_{i}\right)=0$. Since $\mathscr{R}\left(Z \wedge Z_{i}\right)=\mathscr{R}\left(Z \wedge Z_{i}^{\prime}\right)+$ $\mathscr{R}\left(Z \wedge Z_{i}^{\prime \prime}\right)=0$ we will have in the Gauss equation $\left(\alpha\left(Z_{i}, Z_{i}\right), \alpha(Z, Z)\right)=0$. Because $\alpha\left(Z_{i}, Z_{i}\right)$ is orthogonal to $\xi_{2}$, we have $\alpha(Z, Z)$ orthogonal to $\xi_{1}$. Now, writing the Gauss equation for the sectional curvature of a plane spanned by $X \in T_{x} O_{1}$ and $Z \in T_{x} O_{2}$ we get

$$
0=\left\langle A_{\xi_{1}} X, X\right\rangle\left\langle A_{\xi_{1}} Z, Z\right\rangle-\left(\left\langle A_{\xi_{1}} Z, X\right\rangle\right)^{2}=-\left(\left\langle A_{\xi_{1}} Z, X\right\rangle\right)^{2} .
$$

This together with (3.3) implies $A_{\xi_{1}} Z=0$. Thus, range $A_{\xi_{1}} \subset T_{x} O_{1}$ and $A_{\xi_{1}} Z=0$, as we claimed.

Remark. It follows from the proof of Lemma (3.1) that when $\left.\mathscr{R}\right|_{T_{x}}\left(O_{1}\right) \neq 0$, there exists a vector $Y \in T_{x} O_{1}$ such that $\xi_{1}=\alpha(Y, Y) /\|\alpha(Y, Y)\|$. This is obvious if $\left.R\right|_{T_{x}}\left(O_{2}\right) \neq 0$. If not, take $Y$ belonging to $\operatorname{ker} A_{\xi_{2}}\left(\operatorname{rank} A_{\xi_{2}}=1\right)$ and to $T_{x} O_{1}$. There exists such $Y$ such that $\alpha(Y, Y) \neq 0$ because $\left.\mathscr{R}\right|_{T_{x}\left(O_{1}\right)} \neq 0$. Then, $\alpha(Y, Z)=0$ because $\left\langle\alpha(Y, Z), \xi_{1}\right\rangle=\left\langle A_{\xi_{1}} Z, Y\right\rangle=0$ and $\left\langle\alpha(Y, Z), \xi_{2}\right\rangle$ $=\left\langle A_{\xi_{2}} Y, Z\right\rangle=0$. Since the sectional curvature $K(Y, Z)=0$, by the Gauss equation, $\alpha(Y, Y)$ is orthogonal to $\alpha(Z, Z)$. Since $\xi_{1}$ is orthogonal to $\alpha(Z, Z), \xi_{1}$ can be taken as $\alpha(Y, Y)$ normalized.

Now, we start the proof of Theorem 1. Let $x$ be a point in $M$ such that $\nu(x)=0$. By (2.3) $M$ is locally a product over the soul and then we can apply Lemma (3.1) where $\operatorname{dim} U=n, T_{x} O_{1}=T_{x} A$ and $T_{x} O_{2}=T_{x}^{\perp} A$. It follows that the sectional curvatures along planes tangent to $A$ are given by the eigenvalues of the operator $A_{\xi_{1}}$ which is nonsingular on $T_{x} A$, since $\operatorname{dim} U=n$.

Let $\bar{f}=\left.f\right|_{A}: A \rightarrow \mathbb{R}^{n+2}$ be the isometric immersion $f$ restricted to the soul. Since $A$ is a totally geodesic submanifold of $M$, the first normal space of $\bar{f}$, namely, the subspace of the normal space where the second fundamental form lies (see [E, p. 334] for definition), is at most two dimensional. We can easily generalize to $\bar{f}$, Theorems (2.2) and (2.3) of [B-M $\left.\mathrm{M}_{1}\right]$, since they depend only on the fact that the second fundamental form lies in a two-dimensional plane. The Lemma (3.1) implies that there is a point $p \in A$ such that all the sectional curvatures along planes tangent to $A$ at $p$ are positive. Hence [B-M $\left.\mathrm{M}_{1}\right]$ implies, if $m \geq 3$, where $m$ is the dimension of the soul, that $A$ and consequently $M$, are simply connected and $A$ is homeomorphic to a sphere $S^{m}$. Also, if $m=2$, $A$ has to be a homeomorphic either to a sphere $S^{2}$ or to a Real Projective Space $\mathbb{R} P^{2}$.

If $M$ is simply connected, then by (2.2) we have that $M$ is a product $A^{m} \times$ $\mathscr{P}^{n-m}$. If $n-m>1$, it follows by $\left[\mathrm{M}_{1}\right]$ that either $f$ is a product of hypersurface immersions or $f$ takes a complete geodesic into a straight line. In the latter case, this geodesic is a line (in the sense that each segment realizes the distance between its endpoints), hence it must split off isometrically (cf. 
[T]). This would imply that $f$ is at least 1-cylindrical which contradicts our assumption. Then $f$ is a product of hypersurface immersions.

The only thing we need to prove now is that if $n \geq 4$, the soul $A$ cannot be homeomorphic to $\mathbb{R} \mathscr{P}^{2}$. Suppose it is and consider the universal covering $\widetilde{M}$ of $M$. Let us consider $\widetilde{A}$, the soul of $\widetilde{M}$, which is homeomorphic to $S^{2}$ and the immersion $\tilde{f}=P \circ f$ where $P$ is the covering map. Since $n-2 \geq 2$ by $\left[\mathrm{M}_{1}\right], \tilde{f}$ will be a product of hypersurface immersions and then if $\tilde{X} \in$ $T S^{2}$ and $\widetilde{Y} \in\left(T S^{2}\right)^{\perp}, \tilde{\alpha}(\tilde{X}, \widetilde{Y})=0(\tilde{\alpha}$ is the second fundamental form of $\tilde{f}$ ). This implies that $f$ is reducible along $A$. By Proposition (2.5) $M$ is a product over the soul and again because $n-2 \geq 2$, we would have $A$, homeomorphic to $\mathbb{R} P^{2}$, isometrically immersed in codimension 1 , which is the required contradiction.

\section{Proof of Theorem 2}

To prove this theorem, we will use the Fundamental Existence and Uniqueness Theorem for submanifolds (see [C, p. 48]). First, we will prove (ii) of the theorem and then (i) will follow easily using the same arguments. Following Moore's argument in $\left[\mathrm{M}_{2}\right]$, for each $x \in M$, we will take a neighborhood $U \subset M$ of $x$ and on the tube $E=U \times \mathbb{R}^{2}$ contained in the normal bundle, consider differentiable sections $\xi_{1}, \xi_{2}$ such that if we define a second fundamental form $\alpha_{t}: T U \times T U \rightarrow E$ by

$$
\alpha_{t}(X, Y)=\left\langle\alpha(X, Y), \xi_{1}\right\rangle \xi_{1}+t\left\langle\alpha(X, Y), \xi_{2}\right\rangle \xi_{2}
$$

and a normal connection $\nabla_{t}^{\perp}: T U \times E \rightarrow E$ by $\nabla_{t}^{\perp} \xi_{\alpha}=t \nabla^{\perp} \xi_{\alpha}$ for $\alpha=1,2$, $\left(E, \alpha_{\xi}, \nabla_{t}^{\perp}\right)$ will satisfy the Gauss equation

$$
\langle R(X, Y) Z, W\rangle=\left\langle\alpha_{t}(X, W), \alpha_{t}(Y, Z)\right\rangle-\left\langle\alpha_{t}(X, Z), \alpha_{t}(Y, W)\right\rangle
$$

the Codazzi equation

$$
\begin{aligned}
& \nabla_{t_{X}}^{\perp} \alpha(Y, Z)-\alpha_{t}\left(\nabla_{X} Y, Z\right)-\alpha_{t}\left(Y, \nabla_{X} Z\right) \\
& \quad=\nabla_{t_{Y}}^{\perp} \alpha(X, Z)-\alpha_{t}\left(\nabla_{Y} X, Z\right)-\alpha_{t}\left(X, \nabla_{Y} Z\right)
\end{aligned}
$$

and the Ricci equation

$$
\left\langle R_{t}^{\perp}(X, Y) \xi, \eta\right\rangle=\left\langle\left(A_{\xi} \circ A_{\eta}-A_{\eta} A_{\xi}\right)(X), Y\right\rangle
$$

for all $X, Y, Z, W$ in $T U, \xi, \eta$ in the normal space and for $0 \leq t \leq 1$ in such way that if $\left\{f_{t}\right\}$ denotes the family of isometric immersions, we will have $f_{0}(U)$ lying in a hyperplane of $\mathbb{R}^{n+2}$. Since $M$ is simply connected, and $\alpha_{t}$ will be globally defined on $T M \times T M$, the family $\left\{f_{t}\right\}$ is also globally defined.

In order to construct $E$ and $\left\{\xi_{1}, \xi_{2}\right\}$ as above we will prove the lemmas below. Given $x \in M, T_{x} M=T_{x} M_{1} \oplus \mathbb{R}$, we will always denote by $Z$ the tangent vector belonging to $\mathbb{R}$.

(4.1) Lemma. With the same hypothesis of the theorem, if a point $x \in M$, $\alpha(Z, Z)(x) \neq 0$, then there is an orthonormal frame $\left\{\xi_{1}, \xi_{2}\right\}$ in the normal space, such that $A_{\xi_{1}}\left(T_{x} M_{1}\right) \subseteq T_{x} M_{1}, A_{\xi_{1}}(Z)=0$ and $\operatorname{rank} A_{\xi_{2}}=1$.

Proof. It follows easily from Lemma (3.1)(b).

We want to observe that, when $\alpha(Z, Z)$ is nonnull in a neighborhood $V$ of $x, \alpha(Z, Z)$ will define a differentiable normal vector field on $V$. Since 
$\xi_{2}$ can be taken as $\alpha(Z, Z)$ normalized, because $\alpha(Z, Z)$ is orthogonal to $\xi_{1}, \xi_{2}$ is also a differentiable normal vector field on $V$ implying that $\xi_{1}$ is a differentiable normal vector field on $V$ too. Because $\operatorname{rank} A_{\xi_{2}}=1$, the operator $A_{\xi_{2}}$ has eigenvalues with constant multiplicity on $V$; this implies, by $\left[\mathrm{N}_{0}\right]$, that its eigenvectors are differentiable vector fields on $V$. Consequently, their orthogonal projections onto $T_{x} M_{1}$, are differentiable vector fields on $V$. Then, for each $x \in V$, we can take differentiably the subspace $W$ of $T_{x} M_{1}$ given by the orthogonal projection of the kernel of $A_{\xi_{2}}$ onto $T_{x} M_{1}$, i.e.,

$$
W=\left\{Y \in T_{x} M_{1} / A_{\xi_{2}}(Y)=0\right\} .
$$

Since $\operatorname{rank} A_{\xi_{2}}=1$, we have that $W$ is at least $(n-2)$-dimensional.

(4.2) Lemma. With the notations above we have

(i) if $Y \in W, \nabla \frac{1}{Y} \xi_{1}=\nabla_{Y}^{\frac{1}{Y}} \xi_{2}=0$,

(ii) if $\lambda$ is an eigenvalue of $A_{\xi_{1}}$ at $x, \lambda$ is constant along the segment of the geodesic $\gamma(t)=\exp _{x} t Z$ contained in $V$.

Proof. If $\operatorname{dim} W=n-1,\left.A_{\xi_{2}}\right|_{T_{x} M_{1}}$ is identically null and then $A_{\xi_{1}}$ and $A_{\xi_{2}}$ are diagonalized by the same basis. This implies that the normal curvature $\mathscr{R}^{\perp}$ is zero and the lemma is obvious.

Now suppose $\operatorname{dim} W=n-2$. By Lemma (4.1) we have $\left\langle\alpha(Y, Z), \xi_{1}\right\rangle=$ 0 and $\left\langle\alpha(Y, Z), \xi_{2}\right\rangle=0$ whenever $Y \in W$. Denoting by $X$ the vector generating $T_{x} M_{1} \cap W^{\perp}$, we can apply the Codazzi equation to $X, Y, Z$ and $\xi_{1}$ to get

$$
\begin{aligned}
& \left\langle\nabla_{X}^{\perp} \alpha(Y, Z), \xi_{1}\right\rangle-\left\langle\alpha\left(\nabla_{X} Y, Z\right), \xi_{1}\right\rangle-\left\langle\alpha\left(Y, \nabla_{X} Z\right), \xi_{1}\right\rangle \\
& \quad=\left\langle\nabla_{Y}^{\perp} \alpha(X, Z), \xi_{1}\right\rangle-\left\langle\alpha\left(\nabla_{Y} X, Z\right), \xi_{1}\right\rangle-\left\langle\alpha\left(X, \nabla_{Y} Z\right), \xi_{1}\right\rangle .
\end{aligned}
$$

Observe that the left-hand side is equal to zero, since $\alpha(Y, Z)=0, \nabla_{X} Y$ and $A_{\xi_{1}}\left(\nabla_{X} Y\right)$ belong to $T_{X} M_{1}$ and $\nabla_{X} Z=0$. The same reasons together with $\left\langle\alpha(X, Z), \xi_{1}\right\rangle=0$, imply that the right-hand side is equal to

$$
\left\langle\nabla_{Y}^{\perp} \alpha(X, Z), \xi_{1}\right\rangle=-\left\langle\alpha(X, Z), \nabla_{Y}^{\perp} \xi_{1}\right\rangle .
$$

Now the assumption $\operatorname{dim} W=n-2$ implies $\left\langle\alpha(X, Z), \xi_{2}\right\rangle \neq 0$. In fact, since $\operatorname{rank} A_{\xi_{2}}=1$, we have $A_{\xi_{2}}(X)=c A_{\xi_{2}}(Z)$ for some $c \neq 0$. Then

$$
\left\langle\alpha(X, Z), \xi_{2}\right\rangle=\left\langle A_{\xi_{2}} X, Z\right\rangle=c\left\langle A_{\xi_{2}} Z, Z\right\rangle=c\|\alpha(Z, Z)\| \neq 0 .
$$

Therefore $\left\langle\nabla_{Y}^{\frac{1}{Y}} \xi_{1}, \xi_{2}\right\rangle=0$ implying $\nabla_{Y}^{\frac{1}{Y}} \xi_{1}=0$. Expanding $Y\left\langle\xi_{1}, \xi_{2}\right\rangle$ we get $\nabla_{Y}^{\frac{1}{Y}} \xi_{2}=0$.

To prove the second part of the lemma, let us consider the orthonormal basis $\left\{X_{1}, \ldots, X_{n-1}\right\}$ of $T_{x} M_{1}$ diagonalizing $A_{\xi_{1}} \mid T_{x} M_{1}$. From the Codazzi equation applied to $Z, X_{i}$ and $\xi_{1}$ we have

$$
\begin{gathered}
Z\left\langle\alpha\left(X_{i}, X_{i}\right), \xi_{1}\right\rangle-\left\langle\alpha\left(X_{i}, X_{i}\right), \nabla_{Z}^{\frac{1}{Z}} \xi_{1}\right\rangle-2\left(\left\langle\alpha\left(\nabla_{Z} X_{i}, X_{i}\right), \xi_{1}\right\rangle\right) \\
=X_{i}\left\langle\alpha\left(Z, X_{i}\right), \xi_{1}\right\rangle-\left\langle\alpha\left(Z, X_{i}\right), \nabla_{X_{i}}^{\perp} \xi_{1}\right\rangle \\
\quad-\left\langle\alpha\left(\nabla_{X_{i}} Z, X_{i}\right), \xi_{1}\right\rangle-\left\langle\alpha\left(Z, \nabla_{X_{i}} X_{i}\right), \xi_{1}\right\rangle .
\end{gathered}
$$

Now, we show that some terms in the above equation are null and we calculate the others. Since $\left.A_{\xi_{2}}\right|_{W}=0$ and $\nabla_{Z} \frac{1}{Z} \xi_{1}$ is parallel to $\xi_{2}$, writing $X_{i}$ as a linear combination of $X$ and vectors belonging to a basis of $W$, we get

$$
\left\langle\alpha\left(X_{i}, X_{i}\right), \nabla_{Z}^{\perp} \xi_{1}\right\rangle=\left\langle X_{i}, X\right\rangle^{2}\left\langle\alpha(X, X), \nabla_{Z}^{\frac{1}{Z}} \xi_{1}\right\rangle .
$$


Because $X_{i}$ is an eigenvector of $A_{\xi_{1}}$ we have

$$
\left\langle\alpha\left(\nabla_{Z} X_{i}, X_{i}\right), \xi_{1}\right\rangle=\left\langle\nabla_{Z} X_{i}, X_{i}\right\rangle\left\langle\alpha\left(X_{i}, X_{i}\right), \xi_{1}\right\rangle .
$$

But $\left\langle\nabla_{Z} X_{i}, X_{i}\right\rangle=0$ since $X_{i}$ is of unitary length. On the right-hand side, the first and the last terms are clearly null since $A_{\xi_{1}}(Z)=0$. The third one is also null because $X_{i}$ is an eigenvector of $A_{\xi_{1}}$ and then

$$
\left\langle\alpha\left(\nabla_{X_{i}} Z, X_{i}\right), \xi_{1}\right\rangle=\left\langle\nabla_{X_{i}} Z, X_{i}\right\rangle\left\langle\alpha\left(X_{i}, X_{i}\right) \xi_{1}\right\rangle .
$$

But $\left\langle\nabla_{X_{i}} Z, X_{i}\right\rangle=0$ since $M$ is a product and $X_{i} \in T_{x} M_{1}$. Now, we calculate the second term writing again $X_{i}$ as a linear combination of $X$ and vectors belonging to a basis of $W$. Using the facts that $\left.A_{\xi_{2}}\right|_{W}=0$ and $\nabla_{Y} \frac{1}{Y} \xi_{2}=0$, we have

$$
\left\langle\alpha\left(Z, X_{i}\right), \nabla_{X_{i}}^{\perp} \xi_{1}\right\rangle=\left\langle X_{i}, X\right\rangle^{2}\left\langle\alpha(Z, X), \nabla_{X}^{\perp} \xi_{1}\right\rangle .
$$

Therefore, (4.3) is equivalent to

$$
\begin{gathered}
Z\left\langle\alpha\left(X_{i}, X_{i}\right), \xi_{1}\right\rangle-\left\langle X_{i}, X\right\rangle^{2}\left\langle\alpha(X, X), \nabla_{Z}^{\frac{1}{Z}} \xi_{1}\right\rangle \\
\quad=-\left\langle X_{i}, X\right\rangle^{2}\left\langle\alpha(Z, X), \nabla_{X}^{\perp} \xi_{1}\right) .
\end{gathered}
$$

Therefore, to prove (ii) of the lemma is equivalent to proving that

$$
\left\langle\alpha(X, X), \nabla_{Z}^{\perp} \xi_{1}\right\rangle=\left\langle\alpha(Z, X), \nabla_{X}^{\perp} \xi_{1}\right\rangle,
$$

which is equivalent to

$$
\left\langle\alpha(X, X), \xi_{2}\right\rangle\left\langle\nabla_{Z}^{\perp} \xi_{1}, \xi_{2}\right\rangle=\left\langle\alpha(Z, X), \xi_{2}\right\rangle\left\langle\nabla_{X}^{\perp} \xi_{1}, \xi_{2}\right\rangle .
$$

For that, consider the orthonormal basis $\left\{Z_{1}, Z_{2}, \ldots, Z_{n}\right\}$ diagonalizing the operator $A_{\xi_{2}}$ such that $A_{\xi_{2}}\left(Z_{i}\right)=0, i \geq 2$. We can consider $Z_{i} \in W \subset T_{x} M_{1}$, for all $i \geq 3$. Applying the Codazzi equation to $Z_{2}, Z_{i}(i \geq 3)$ and $\xi_{2}$, we have

$$
\begin{aligned}
& \left\langle\nabla_{Z_{2}}^{\perp} \alpha\left(Z_{i}, Z_{i}\right), \xi_{2}\right\rangle-2\left\langle\alpha\left(\nabla_{Z_{2}} Z_{i}, Z_{i}\right), \xi_{2}\right\rangle \\
& \quad=\left\langle\nabla_{Z_{i}}^{1} \alpha\left(Z_{2}, Z_{i}\right), \xi_{2}\right\rangle-\left\langle\alpha\left(\nabla_{Z_{i}} Z_{2}, Z_{i}\right), \xi_{2}\right\rangle-\left\langle\alpha\left(Z_{2}, \nabla_{Z_{i}} Z_{i}\right), \xi_{2}\right\rangle .
\end{aligned}
$$

On the left-hand side, because $A_{\xi_{2}} Z_{i}=0$, we will have only

$$
\left\langle\nabla_{Z_{2}}^{\perp} \alpha\left(Z_{i}, Z_{i}\right), \xi_{2}\right\rangle=-\left\langle\alpha\left(Z_{i}, Z_{i}\right), \nabla_{Z_{2}}^{\perp} \xi_{2}\right\rangle .
$$

Since $i \geq 3$ and $Z_{i} \in W$, from (i) of this lemma we have $\nabla \frac{1}{Z_{i}} \xi_{2}=0$. Again, because $A_{\xi_{2}} Z_{2}=A_{\xi_{2}} Z_{i}=0$, the right-hand side is equal to zero. Then (4.5) becomes

$$
\left\langle\alpha\left(Z_{i}, Z_{i}\right), \xi_{1}\right\rangle\left\langle\nabla \frac{1}{Z_{2}} \xi_{2}, \xi_{1}\right\rangle=0 .
$$

If there is $i$ such that $\left\langle\alpha\left(Z_{i}, Z_{i}\right), \xi_{1}\right\rangle \neq 0$, we have $\left\langle\nabla_{Z_{2}}^{\frac{1}{2}} \xi_{2}, \xi_{1}\right\rangle=0$. If $\left\langle\alpha\left(Z_{i}, Z_{i}\right), \xi_{1}\right\rangle=0$ for all $i \geq 3$, we have $\left.A_{\xi_{1}}\right|_{W}=0$ and then $X$ is an eigenvector of $A_{\xi_{1}}$. Writing the Codazzi equation for $Z_{2}, X, Z$ and $\xi_{1}$ we will have

$$
\begin{aligned}
& \left\langle\nabla_{Z_{2}}^{\perp} \alpha(X, Z), \xi_{1}\right\rangle-\left\langle\alpha\left(\nabla_{Z_{2}} X, Z\right), \xi_{1}\right\rangle-\left\langle\alpha\left(X, \nabla_{Z_{2}} Z\right), \xi_{1}\right\rangle \\
& \quad=\left\langle\nabla_{X}^{\perp} \alpha\left(Z_{2}, Z\right), \xi_{1}\right\rangle-\left\langle\alpha\left(\nabla_{X} Z_{2}, Z\right), \xi_{1}\right\rangle-\left\langle\alpha\left(Z_{2}, \nabla_{X} Z\right), \xi_{1}\right\rangle .
\end{aligned}
$$

Since $X$ is the only eigenvector of $A_{\xi_{1}}$ with nonnull eigenvalue, $Z_{2} \in$ $\operatorname{span}\{X, Z\}$ and $\left\langle\nabla_{X} X, Z\right\rangle=0$, the only term we will have is

$$
\left\langle\nabla_{Z_{2}}^{\perp} \alpha(X, Z), \xi_{1}\right\rangle=-\left\langle\alpha(X, Z), \nabla_{Z_{2}}^{\perp} \xi_{1}\right\rangle
$$


which will be zero too. Since we are considering $\left\langle\alpha(X, Z), \xi_{2}\right\rangle \neq 0$ (otherwise $\operatorname{dim} W=n-1)$ we have again $\nabla \frac{1}{Z_{2}} \xi_{1}=\nabla_{Z_{2}} \xi_{2}=0$. This fact will imply (4.4). In fact, write $Z_{2}=a X+b Z$,

$$
\begin{gathered}
A_{\xi_{2}}\left(Z_{2}\right)=0 \Rightarrow a\left\langle\alpha(X, X), \xi_{2}\right\rangle=-b\left\langle\alpha(X, Z), \xi_{2}\right\rangle, \\
\nabla_{Z_{2}}^{\perp} \xi_{1}=0 \Rightarrow a \nabla_{X}^{\perp} \xi_{1}=-b \nabla_{Z}^{\perp} \xi_{1} .
\end{gathered}
$$

Therefore

$$
b\left\langle\alpha(X, Z), \xi_{2}\right\rangle \nabla_{X}^{\perp} \xi_{1}=b\left\langle\alpha(X, X), \xi_{2}\right\rangle \nabla_{Z}^{\perp} \xi_{1} .
$$

Since we are supposing $b \neq 0\left[\left(b=0 \Rightarrow Z_{2} \in T_{x} M_{1} \Rightarrow \operatorname{dim} W=n-1\right)\right]$, (4.7) implies (4.5) taking product with $\xi_{2}$.

(4.8) Lemma. With the notation above, if $Y \in W$ and $X$ is orthogonal to $W$, $Y$ and $X$ are parallel along the segment of geodesic $\gamma(t)=\exp _{x} t Z$ contained in $V$.

Proof. If $M_{1}$ is two dimensional we consider isothermal parameters $\left(x_{1}, x_{2}\right)$ defined on $V$ such that $\partial / \partial x_{1}=\lambda_{1} X$ and $\partial / \partial x_{2}=\lambda_{2} Y$. If $M_{1}$ is at least three dimensional then $\operatorname{dim} W \geq 2$. Consider $Y_{1}, Y_{2} \in W$ and the Codazzi equation for $Y_{1}, Y_{2}, X$ and $\xi_{2}$. We have

$$
\begin{aligned}
& \left\langle\nabla_{Y_{1}}^{\perp} \alpha\left(Y_{2}, X\right), \xi_{2}\right\rangle-\left\langle\alpha\left(\nabla_{Y_{1}} Y_{2}, X\right), \xi_{2}\right\rangle-\left\langle\alpha\left(Y_{2}, \nabla_{Y_{1}} X\right), \xi_{2}\right\rangle \\
& \quad=\left\langle\nabla_{Y_{2}}^{\perp} \alpha\left(Y_{1}, X\right), \xi_{2}\right\rangle-\left\langle\alpha\left(\nabla_{Y_{2}} Y_{1}, X\right), \xi_{2}\right\rangle-\left\langle\alpha\left(Y_{1}, \nabla_{Y_{2}} X\right), \xi_{2}\right\rangle .
\end{aligned}
$$

Since $A_{\xi_{2}}\left(Y_{i}\right)=0$ and $\nabla_{Y_{i}}^{\perp} \xi_{2}=0$ for $i=1,2$, we will have

$$
\left\langle\nabla_{Y_{1}} Y_{2}, X\right\rangle\left\langle\alpha(X, X), \xi_{2}\right\rangle=\left\langle\nabla_{Y_{2}} Y_{1}, X\right\rangle\left\langle\alpha(X, X), \xi_{2}\right\rangle \text {. }
$$

Since $\left\langle\alpha(X, X), \xi_{2}\right\rangle \neq 0$ (otherwise $\operatorname{dim} W=n-1$ which implies $\mathscr{R}^{\perp}=0$ and then the lemma is obvious) we have $\left\langle\left[Y_{1}, Y_{2}\right], X\right\rangle=0$ and then $W$ is integrable. By Frobenius we can consider local coordinates $\left(x_{1}, \ldots, x_{n-1}, t\right)$ defined on $V$ such that $\partial / \partial x_{1}=\lambda_{1} X, \partial / \partial x_{i}=\lambda_{i} Y_{i}$ for $Y_{i} \in W$ and $\partial / \partial t=$ $\lambda_{n} Z$. Since $\left[\partial / \partial x_{i}, \partial / \partial t\right]=0$, we have

$$
\nabla_{\partial / \partial x_{i}} \frac{\partial}{\partial_{t}}-\nabla_{\partial / \partial x_{t}} \frac{\partial}{\partial x_{i}}=\nabla_{\lambda_{i} Y_{i}} \lambda_{n} Z-\nabla_{\lambda_{n} Z} \lambda_{i} Y_{i}=0
$$

Therefore

$$
\lambda_{i} Y_{i}\left(\lambda_{n}\right) Z+\lambda_{i} \lambda_{n} \nabla_{Y_{i}} Z-\lambda_{n} Z\left(\lambda_{i}\right) Y_{i}-\lambda_{n} \lambda_{i} \nabla_{Z} Y_{i}=0 .
$$

Since $\nabla_{Y_{i}} Z=0$ (because $M$ is a product) and $\nabla_{Z} Y_{i}$ is orthogonal to $Z$ and $Y_{i}$, it follows that $\nabla_{Z} Y_{i}=0$. The same proof implies $\nabla_{Z} X=0$.

Now, we can start the proof of Theorem 2. Given $x \in M$, we consider the geodesic $\gamma(t)=\exp _{x} t Z$. We want to prove that for each point $p \in \gamma$, there is a neighborhood $U$ of $p$ such that we have differentiable normal vector fields $\xi_{1}$ and $\xi_{2}$ satisfying Lemmas (4.1) (rank $A_{\xi_{2}}$ does not need to be 1 but 0 or $1),(4.2)$ and (4.3).

First, we claim that there is a point $y \in \gamma$ such that $\alpha(Z, Z)(y) \neq 0$ because if not, $f$ would take a complete geodesic to a straight line $\mathbb{R}^{n+2}$ contradicting our assumption. As we have already observed, in a neighborhood $U$ of $y$, we get $\xi_{1}$ and $\xi_{2}$ with the desired properties. 
If $\alpha(Z, Z)=0$ at an isolated point of $\gamma$, denoted by $\gamma\left(t_{0}\right)$, we have $\xi_{1}$ and $\xi_{2}$ defined on its right and $\bar{\xi}_{1}$ and $\bar{\xi}_{2}$ defined on its left. We claim that $\xi_{1}=\bar{\xi}_{1}$ and $\xi_{2}=\bar{\xi}_{2}$ (defined by limit) at the point $\gamma\left(t_{0}\right)$. In fact, since for each point $p \in \gamma$ there is a two-plane $\sigma \subset T_{p} M_{1}$ such that $k(\sigma)>0$, for $t$ close to $t_{0}$ and $t>t_{0}$, there exists $Y \in T_{\gamma(t)} M_{1}$ such that $\xi_{1}$ is $\alpha(Y, Y)$ normalized. For $t$ close to $t_{0}$ and $t<t_{0}$, there also exists $\bar{Y} \in T_{\gamma(t)} M_{1}$ such that $\bar{\xi}_{1}$ is $\alpha(\bar{Y}, \bar{Y})$ normalized. Lemma (4.2)(ii) implies they are defined of the same manner, by limit, at $\gamma\left(t_{0}\right)$. Now, we define vector $Y$ for $t \leq t_{0}$ and $\bar{Y}$ for $t \geq t_{0}$ by parallel translation along the geodesic $\gamma$ (see Lemma (4.8)). Since rank $A_{\xi_{2}} \leq 1$ and rank $A_{\bar{\xi}_{2}} \leq 1$, the curvature operator $\mathscr{R}$ at $\gamma\left(t_{0}\right)$ is given by

$$
\mathscr{R}=A_{\bar{\xi}_{1}} \wedge A_{\bar{\xi}_{1}}=A_{\xi_{1}} \wedge A_{\xi_{1}} .
$$

Writing $\bar{\xi}_{1}$ as a linear combination of $\xi_{1}$ and $\xi_{2}$, a straightforward calculation for one nonnull sectional curvature implies $\xi_{1}\left(t_{0}\right)= \pm \bar{\xi}_{1}\left(t_{0}\right)$. Therefore $\xi_{1}=\bar{\xi}_{1}$ at $\gamma\left(t_{0}\right)$ otherwise $Y$ and $\bar{Y}$ would span a two-plane whose sectional curvature would be negative. Then

$$
\xi_{1}=\bar{\xi}_{1}=\frac{\alpha(Y, Y)}{\|\alpha(\bar{Y}, \bar{Y})\|}=\frac{\alpha(\bar{Y}, \bar{Y})}{\|\alpha(\bar{Y}, \bar{Y})\|}
$$

and this also proves the differentiability of $\xi_{1}$ in a neighborhood of $\gamma\left(t_{0}\right)$.

If $\alpha(Z, Z)=0$ in a whole interval denoted by $\left[t_{1}, t_{2}\right], \xi_{1}\left(t_{1}\right), \xi_{2}\left(t_{1}\right), \bar{\xi}_{1}\left(t_{2}\right)$ and $\bar{\xi}_{2}\left(t_{2}\right)$ are defined by limit since $\gamma\left(t_{1}\right)$ and $\gamma\left(t_{2}\right)$ are on the boundaries of neighborhoods where $\alpha(Z, Z) \neq 0$. We extend them to the interior of the interval by parallel translation along $\gamma$. First, we show that they have the desired properties. Consider $X_{i}$ an eigenvector of $A_{\xi_{j}}, j=1,2$. From the Codazzi equation we have

$$
\begin{aligned}
Z\left\langle\alpha\left(X_{i}, X_{i}\right), \xi_{j}\right\rangle-\left\langle\alpha\left(X_{i}, X_{i}\right), \nabla_{Z}^{1} \xi_{j}\right\rangle-2\left\langle\alpha\left(\nabla_{Z} X_{i}, X_{i}\right), \xi_{j}\right\rangle \\
=X_{i}\left\langle\alpha\left(Z, X_{i}\right), \xi_{j}\right\rangle-\left\langle\alpha\left(Z, X_{i}\right), \nabla_{X_{i}}^{1} \xi_{j}\right\rangle-\left\langle\alpha\left(\nabla_{X_{i}} Z, X_{i}\right), \xi_{j}\right\rangle \\
\quad-\left\langle\alpha\left(Z, \nabla_{X_{i}} X_{i}\right), \xi_{j}\right\rangle .
\end{aligned}
$$

Since $A_{\xi_{1}}(Z)=0, \nabla_{Z}^{\perp} \xi_{j}=0$ and $\nabla_{X_{i}} Z=0$ we have

$$
Z\left\langle\alpha\left(X_{i}, X_{i}\right), \xi_{j}\right\rangle=0 .
$$

Now, repeating the same argument used above we show that the extensions agree on the interior of the interval. $\xi_{1}\left(t_{1}\right)$ is given by $\alpha(Y, Y)$ normalized, where $Y \in T_{\gamma\left(t_{1}\right)} M_{1}$ and $\xi_{1}\left(t_{2}\right)$ is given by $\alpha(\bar{Y}, \bar{Y})$ normalized, where $\bar{Y} \in T_{\gamma\left(t_{2}\right)} M_{1}$. Because on the interior of the interval $\xi_{2}(t)$ and $\bar{\xi}_{2}(t)$ have the desired properties, $\xi_{1}$ must be given by $\alpha(Y, Y)$ normalized and $\bar{\xi}_{1}$ by $\alpha(\bar{Y}, \bar{Y})$ normalized, $\operatorname{rank} A_{\xi_{2}} \leq 1$ and $\operatorname{rank} A_{\bar{\xi}_{2}} \leq 1$. Then $\xi_{1}(t)=\bar{\xi}_{2}(t)$, otherwise $Y$ and $\bar{Y}$ would span a plane whose sectional curvature would be negative.

To finish the proof of (ii) in Theorem 2, we have to prove that $\alpha_{t}$ defined previously, satisfies Gauss, Codazzi and Ricci equations. The Gauss equation will follow from (3.2). The Codazzi and Ricci equations follow easily if $0<$ $t \leq 1$. For $t=0, \alpha_{t}(X, Y)=\left\langle\alpha(X, Y), \xi_{1}\right\rangle \xi_{1}$, which implies that $f_{0}$ will be an isometric immersion in a hyperplane of $\mathbb{R}^{n+2}$. Therefore, we need to prove 
only the Codazzi equation. This will follow from the Codazzi equation for the given isometric immersion $f$, remembering that for $Y \in W$,

$$
\begin{aligned}
& \left\langle\nabla_{Y}^{\perp} \alpha\left(X_{1}, X_{2}\right), \xi_{1}\right\rangle=Y\left\langle\alpha\left(X_{1}, X_{2}\right), \xi_{1}\right\rangle \text { since } \nabla_{Y}^{\perp} \xi_{1}=0 \text {, } \\
& \left\langle\nabla \frac{1}{X_{1}} \alpha\left(Y, X_{2}\right), \xi_{1}\right\rangle=X_{1}\left\langle\alpha\left(Y, X_{2}\right), \xi_{1}\right\rangle \text { since } A_{\xi_{2}}(Y)=0 \text {, }
\end{aligned}
$$

for each $X_{1}, X_{2}$ tangent to $M$. Hence, we are reduced to prove them for $X$ and $Z, X$ orthogonal to $W$. The first of them is

$$
\begin{aligned}
& X\left\langle\alpha(Z, Z), \xi_{1}\right\rangle-2\left\langle\alpha\left(\nabla_{X} Z, Z\right), \xi_{1}\right\rangle=0 \\
& \quad=Z\left\langle\alpha(X, Z), \xi_{1}\right\rangle-\left\langle\alpha\left(\nabla_{Z} X, Z\right), \xi_{1}\right\rangle-\left\langle\alpha\left(X, \nabla_{Z} Z\right), \xi_{1}\right\rangle
\end{aligned}
$$

because $A_{\xi_{1}}(Z)=0$ and $\nabla_{Z} Z=0$. The other equation will be

$$
\begin{aligned}
& Z\left\langle\alpha(X, X), \xi_{1}\right\rangle-2\left\langle\alpha\left(\nabla_{Z} X, X\right), \xi_{1}\right\rangle=Z\left\langle\alpha(X, X), \xi_{1}\right\rangle \\
& =X\left\langle\alpha(Z, X), \xi_{1}\right\rangle-\left\langle\alpha\left(\nabla_{X} Z, X\right), \xi_{1}\right\rangle-\left\langle\alpha\left(Z, \nabla_{X} X\right), \xi_{1}\right\rangle=0
\end{aligned}
$$

because $A_{\xi_{1}}(Z)=0$ and $\nabla_{X} Z=\nabla_{Z} X=0$. We can see that the only term left, $Z\left\langle\alpha(X, X), \xi_{1}\right\rangle$ is zero from the Codazzi equation for $f$ which has only one more term on the left-hand side, i.e., $\left\langle\alpha(X, X), \nabla \frac{1}{Z} \xi_{1}\right\rangle$ and only one more term on the right-hand side $\left\langle\alpha(Z, X), \nabla_{Z}^{\perp} \xi_{1}\right\rangle$ which are equal by (4.4).

Now, part (i) of the theorem is easily proved. For each $\bar{x} \in M_{1}$, we consider $x=(\bar{x}, t) \in M_{1} \times \mathbb{R}$, such that $\alpha(Z, Z)(x) \neq 0$. For a neighborhood $U$ of $x$, which is $\bar{U} \times I\left(\bar{U}\right.$ is a neighborhood of $x$ in $M_{1}$ and $I$ an interval of $\mathbb{R})$ we have a differentiable normal vector $\xi_{1}$, such that $A_{\xi_{1}}$ satisfies the Gauss and Codazzi equations for an isometric immersion from $\bar{U}$ to $\mathbb{R}^{n}$. Since $M_{1}$ is simply connected, we have the required immersion $g$.

We observe that $f_{0}$, constructed above, is congruent to $g \times i$ since $A_{\xi_{1}}(Z)=$ 0 .

\section{THEOREM 3 AND IMMERSIONS WHICH ARE NOT PRODUCTS}

To prove Theorem 3, by Theorem 1 we have to consider only two cases, namely, when the soul is the circle $S^{1}$ and when $n=3$ and the soul is homeomorphic to $\mathbb{R} P^{2}$. In both cases, the universal covering $M$ of the manifold $M$ will be the product $M_{1} \times \mathbb{R}$.

We will denote by $\tilde{f}=f \circ P \quad(P$ is the covering map $), \tilde{\alpha}$ the second fundamental form of $\tilde{f}$, and $\nu_{\tilde{f}}(\tilde{x})$ the index of relative nullity of $\tilde{f}$ at $\tilde{x} \in \widetilde{M}$. Let us take $\tilde{x} \in \widetilde{M}$ such that $\nu_{\tilde{f}}(\tilde{x})=0$. Following the proof of Lemma (3.1) we see that at a point $x$ where $\nu(x)=0$, there are normal vectors $\xi_{1}$ and $\xi_{2}$ such that $A_{\xi_{1}}\left(T_{x} M_{1}\right)=T_{x} M_{1}, A_{\xi_{1}}(Z)=0$ and $\operatorname{rank} A_{\xi_{2}}=1$ ( $Z$ denotes the tangent vector belonging to $\mathbb{R})$. Since $\mathscr{R}^{\perp}=0$, the same orthonormal basis diagonalizes $A_{\xi_{1}}$ and $A_{\xi_{2}}$. Then $Z_{1}$, eigenvector of $A_{\xi_{2}}$ corresponding to nonnull eigenvalue, has to be tangent either to $M_{1}$ or to $\mathbb{R}$. If $Z_{1} \in T_{x} M_{1}$, $A_{\xi_{2}}(Z)=0$ and this implies that $Z$ is relative nullity vector, which contradicts $\nu_{\tilde{f}}(\tilde{x})=0$. Then $Z_{1}=Z$ and $\tilde{\alpha}(X, Z)=0$ for all $X \in T_{x} M_{1}$. The proof of Theorem 2 in $\left[\mathrm{M}_{1}\right]$ implies $\tilde{\alpha}(X, Z)=0$ for all $X \in T M_{1}$ since no complete geodesic in $\widetilde{M}$ can be taken to a straight line in $\mathbb{R}^{n+2}$. Therefore $f$ is reducible along the soul $A$. By Proposition (2.5) $M$ is a product over the soul and because $\alpha(X, Z)=0, f$ is a product of hypersurface immersions, proving the theorem. 
Now, we show an example of a complete manifold $M^{n}$ with $S^{1}$ as a soul, isometrically immersed in $\mathbb{R}^{n+2}$ which is not a product of immersions. The reader is referred to [A-M, $\S 4]$, for the details.

Let $f_{1}: M_{1}^{n-1} \rightarrow \mathbb{R}^{n}$ be any isometric immersion of codimension one and $f_{2}: S^{1} \rightarrow \mathbb{R}^{2}$ an isometric immersion such that $f_{2}$ is totally geodesic on the interval $I=(-\varepsilon, \varepsilon)$. Now consider $f=f_{1} \times f_{2}: M=M_{1} \times S^{1} \rightarrow \mathbb{R}^{n+2}$. The restriction of the immersion $f$ to $M_{1} \times I$ is given by

$$
(m, t) \mapsto \sum_{i=1}^{n+1} f_{1}^{i}(m) e_{i}+t e_{n+2}
$$

where $\left\{e_{1}, \ldots, e_{n+2}\right\}$ is the standard basis of $\mathbb{R}^{n+2}$.

If $M_{1}$ is compact in $\S 4$ of [A-M], the authors show how to construct an isometric immersion $f$ of $M_{1} \times I$ which is not a product of hypersurface immersions and agrees with $f$ whenever $\varepsilon / 2<|t|<\varepsilon$.

We want to point out that the compactness of $M_{1}$ is necessary to guarantee that equation (7) in [A-M] never vanishes and then $h_{1}$ defined by them is an immersion. We are interested when $M_{1}$ is noncompact. So, we will fix one copy $S^{1}$ of $M$, denote it by $\bar{S}$, and for a positive real number $\sigma$, take the compact subset $B(\bar{S}, \sigma)=\{m \in M / d(m, \bar{S})<\sigma\} \quad(d(m, \bar{S})$ is the distance from $m$ to $\bar{S}$ ). We redefine the immersion $h: M_{1} \times I \rightarrow \mathbb{R}^{n+2}$ by

$$
h(m, t)=\sum_{i=1}^{n-1} f_{1}^{i}(m) e_{i}+f_{1}^{n}(m)\left[X_{n}+\alpha X_{n+1}\right](t)+\gamma(t)
$$

where now $\alpha$ is a smooth function depending on $(m, t)$. For $m \in B(\bar{S})$, $\alpha(m, t)$ satisfies the same conditions as $\alpha$ in [A-M] as a function of $t$, and we require that $\alpha(m, t)$ goes to zero when $m$ is such that $d(m, \bar{S})=\sigma \cdot \gamma$ is as in $[\mathrm{A}-\mathrm{M}]$.

Following the same procedure as in $[\mathrm{A}-\mathrm{M}]$ we can produce an isometric immersion $\bar{f}$ of $M_{1} \times I$ which agrees with $f$ whenever $\varepsilon / 2<|t|<\varepsilon$ and $m \notin B(\bar{S}, \sigma)$.

Piecing $f$ and $\bar{f}$ together we obtain a 2-codimensional isometric immersion of $M_{1} \times S^{1}$ which is not a product of immersions.

Before finishing this paper we would like to say that it would be of interest to construct examples of manifolds having $S^{1}$ as soul in $\mathbb{R}^{n+2}$, which are not product over the soul.

\section{REFERENCES}

[A] S. Alexander, Reducibility of Euclidean immersions of low codimension, J. Differential Geom. 3 (1969), 69-82.

[A-M] S. Alexander and R. Maltz, Isometric immersions of Riemannian products in euclidean space, J. Differential Geom. 11 (1976), 47-57.

[B-M $\left.\mathrm{M}_{1}\right] \mathrm{Y} . \mathrm{Y}$. Baldin and $\mathrm{F}$. Mercuri, Isometric immersions in codimension two with nonnegative curvature, Math. Z. 173 (1980), 111-117.

$\left[\mathrm{B}-\mathrm{M}_{2}\right]$, Codimension two nonorientable submanifolds with nonnegative curvature, Proc. Amer. Math. Soc. 103 (1988), 918-920.

[B-N] Y. Y. Baldin and M. H. Noronha, Some complete manifolds with nonnegative curvature operator, Math. Z. 195 (1987), 383-390. 
[Bi] R. L. Bishop, The holonomy algebra of immersed manifolds of codimension two, J. Differential Geom. 2 (1968), 347-353.

[C] B. Y. Chen, Geometry of submanifolds, Dekker, New York, 1973.

[C-G] J. Cheeger and D. Gromoll, On the structure of complete manifolds of nonnegative curvature, Ann. of Math. (2) 96 (1972), 413-443.

[E] J. Erbacher, Reduction of the codimension of an isometric immersion, J. Differential Geom. 5 (1971), 333-340.

[G-R] M. L Gromov and V. A. Rokhlin, Embeddings and immersions in Riemannian geometry, Uspekhi Mat. Nauk 25 (1976), 3-62.

[H] P. Hartman, On the isometric immersions in Euclidean space of manifolds with nonnegative sectional curvatures. II, Trans. Amer. Math. Soc. 147 (1970), 529-540.

$\left[\mathrm{M}_{1}\right]$ J. D. Moore, Isometric immersions of Riemannian products, J. Differential Geom. 5 (1971), 159-168.

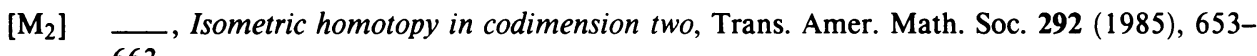
663.

[ $\left.\mathrm{N}_{0}\right] \mathrm{K}$. Nomizu, Characteristic roots and vectors of a differentiable family of symmetric matrices, Linear and Multilinear Algebra 1 (1973), 159-162.

$\left[\mathrm{N}_{1},\right]$ M. H. Noronha, Codimension two complete noncompact submanifolds with nonnegative curvature, Trans. Amer. Math. Soc. 311 (1989), 739-748.

$\left[\mathrm{N}_{2},\right] \quad \ldots$, A splitting theorem for complete manifolds with nonnegative curvature operator, Proc. Amer. Math. Soc. 105 (1989), 979-985.

[O] B. O'Neill, The fundamental equations of a submersion, Michigan Math. J. 13 (1966), 459469.

[T] V. A. Toponogov, Spaces with straight lines, Amer. Math. Soc. Transl. 37 (1964), 287-290.

[W] A. Weinstein, Positively curved n-manifolds in $\mathbb{R}^{n+2}$, J. Differential Geom. 4 (1970), 1-4.

[Y] J. W. Yim, Distance nonincreasing retraction on a complete open manifold of nonnegative sectional curvature, Ann. Global Anal. Geom. 6 (2) (1988).

Departamento de Matemática, IMECC-UNICAMP, 13.081-Campinas, SP, Brasil

Current address: Department of Mathematics, California State University, Northridge, California 91330 